\title{
Efficient Load Balancing Routing Technique for Mobile Ad Hoc Networks
}

\author{
Mahdi Abdulkader Salem \\ Department of Computer Sciences \& Information \\ Technology Sam Higginbottom Institute of Agriculture, \\ Technology \& Sciences, Allahabad India
}

\author{
Raghav Yadav \\ Department of Computer Science \& Information \\ Technology Sam Higginbottom Institute of Agriculture, \\ Technology \& Sciences, Allahabad India
}

\begin{abstract}
The mobile ad hoc network (MANET) is nothing but the wireless connection of mobile nodes which provides the communication and mobility among wireless nodes without the need of any physical infrastructure or centralized devices such as access point or base station. The communication in MANET is done by routing protocols. There are different categories of routing protocols introduced with different goals and objectives for MANETs such as proactive routing protocols (e.g. DSDV), reactive routing protocols (e.g. ADOV), geographic routing protocols (e.g. GRP), hybrid routing protocols etc. There are two important research problems with such routing protocols to address such as efficient load balancing and energy efficiency. In this paper, we are focusing on evaluation and analysis of efficient load balancing protocol design for MANET. Inefficient load balancing technique results in increasing routing overhead, poor packet delivery ratio, and other Quality of Service (QoS) parameters. In literature, there are a number of different methods proposed for improving the performance of routing protocols by efficient load balancing among mobile nodes communication. However, most of the methods suffer from various limitations. In this paper, we propose a novel technique for improved the QoS performance of load balancing approach as well as increasing the network lifetime. Evaluation of Network lifetime is out of scope of this paper.
\end{abstract}

Keywords-AODV; MANET; Load balancing; throughput; packet delivery ratio; routing overhead

\section{INTRODUCTION}

The term MANET (mobile ad hoc network) is defined as the wireless autonomous network in which nodes get connected by wireless links without using any physical infrastructure. MANET is a temporary network. Each node in MANET acts as both data sender or receiver and data forwarder in particular direction selected by routing protocol. All mobile nodes in network move randomly within a specific network area. Battlefield is one of the main area where MANET is widely used. For intercommunication purpose, such kind of networks do not need any extra support in the form of base stations or access points. Therefore, it is a total dynamic and infrastructure-free network. MANET network is nothing but a group of radio devices in which wireless communication is executed without any fixed physical foundation. The communication between the source mobile node and destination mobile node is not direct, but rather is carried by using intermediate nodes according to multi-hop communication approach. Direct communication can only be possible among neighboring nodes.
The mobile nodes in MANET are located randomly and continuously changing their positions in network. Thus, the interconnections among mobile nodes are also changing frequently. Such networks are thus self-organizing and selfconfiguring and one does not require central management for configuration purpose. In MANET, all nodes can communication each other using the wireless links. Due to the characteristics of MANET like allowing access to servies anywhere, anytime ubiquitously without need of any physical devices or platform, it is mainly used in crisis management services, military areas, conference halls, classrooms, etc. Development of multimedia applications like video conferencing and video on demand is possible only because of ad hoc networking developments of MANETs.

The communication in MANETs is possible due to use of routing protocols which help to discover the communication paths, select the paths, forward data on current paths, maintain the routes, and handle frequent changes in routes due to frequent nodes movements. The traditional and existing routing protocols did not addressed the issues related to QoS (Quality of Service). QoS is nothing but the level of performance of particular routing protocol of service providing to network end users. Many real time applications especially multimedia programs having the QoS requirements which must be achieved. The basic aim of QoS solutions is to get the improved deterministic behaviour of network with the objective of delivering the data carried by wireless network rightly, and utilization of network resources should be efficient. However, there is still the research challenge of maintaining the QoS solutions according to end users mobility. Many of the existing routing protocols presented so far for MANET are targeted either at minimizing the data traffic in wireless network or at reducing the number of hops taken to deliver packets.

The main reason of not providing the QoS solutions with existing routing protocols is that they are not designed with load balancing approach to cope with diver's conditions of MANET, mobility, data traffic, etc. For Mobile ad hoc networks, one of the important problem is load balancing. As we discussed earlier in this paper, the existing routing protocols do not have the processing of dealing with load balancing in MANET. Therefore, since the last two decades, a number of methods have been designed for load balancing in routing protocols. Due to emerging application requirements and also for reliable data transfer, load balancing is one of the key research areas in the field of MANETs. In MANETs, task 
finishing in particular is more complex if there is more traffic on mobile nodes with minimized processing capacity. There is no special technique for load sharing. Non-uniform processing or computing power of systems resulted in load imbalance in MANET. Sometimes, certain nodes in the network are overloaded and some nodes are completely idle. The mobile node with better processing capabilities can complete its tasks in quick time. Such nodes are treated that it does not load or less load all the time. Therefore, situation of nodes with less load are keep idle, and requirement of over loaded mobile nodes is objectionable.

Many routing approaches have been developed for load balancing in MANETs. Major research work is carried out by approaching the load balancing problem through congestion estimation and traffic control. Some approaches are used in energy and power metrics for making routing decision for load balancing. Clustering-based approaches also exist. Very few literatures use queue size, hop count, and bandwidth metrics for load balancing in mobile ad hoc networks. However, for MANET, there are two research challenges such as QoS improvement and energy efficiency. These two points were not efficiently addressed by existing methods. Therefore, it becomes a motivation for this research paper to present novel hybrid approach for load balancing in MANET with goal of improving QoS performance and energy efficiency performance.

The main goal of this paper is thus to present novel algorithm for efficient load balancing technique. This proposed method should address both load balancing and energy efficiency parallel. The proposed method has two important features such as method of link estimation proposed for energy efficiency improvement and another is learning of network load balancing in order to achieve the improved QoS solutions. These two contribution points are combined together in order to achieve both energy efficiency and improved QoS performance. However, in this paper, we keep the scope particularly with evaluation of QoS performance of efficient load balancing technique. Energy efficiency is out of scope of this paper. The rest of this paper is organized as follows: section II presents the related work studies over different load balancing techniques with analysis. Section III presents an overview of proposed framework, algorithm steps and design. Section IV presents the simulation studies and results achieved with different network conditions. Finally Section V presents the conclusion and discusses future work.

\section{RELATED WORK}

In [1], the authors Yin and Lin presented the MALB technique which is based on multipath communication load balancing approach. For every discovered path, this protocol iteratively tracking the current traffic rate. The tracking of traffic rate is done for reducing the end-to-end delay performance in network.

In [2], authors present another technique which is based on similar approach presented in [1] for multipath communication based protocol.

In [3], authors $\mathrm{Wu}$ and Harms introduce the communication among the 2 node disjoint routes as the number paths among the nodes on different routes. From the practical analysis and results of this method, it can be seen that increasing the correlation results in increasing end to end delay for two numbers of paths. Therefore, to decrease this end to end delay performance, another approach is introduced in which traffic balancing is done around the least correlated routes.

In [4], [5], [6], different uni path based load balancing techniques are proposed by authors. In [4], various routing metrics are considered. In [5], packet caching approach is adopted. In [6], directional antennas approach is used.

In [7], the authors Zhu and Hassanein propose the new load balancing routing method known as LBAR. This protocol considers the nodal activity for routing metric from the total number of valid routes.

In [8], authors Lee and Riley presented approach for overloaded mobile nodes in which it is presented that such nodes would have freedom to forbidding the extra communications in order to make them load free by solving their overloaded condition. Therefore, every mobile device of MANET having the specific threshold value for making the decision on receipt of RREQ messages. There are number of other articles presented in which comparative study among multi path and single path load balancing techniques is discussed. Practically, the multipath-based load balancing methods providing the various benefits for improving the fault tolerance as well as reliability. However, it is showing that in [9], single path based techniques claim to be more efficient for load balancing.

In [9], author Pearlman, et al. introduced an approach for multipath-based routing method which is efficient if the alternate routes are disjoint. However, this is not simple to achieve in MANETs [10]. In [11], author Ganjali and Keshavarzian proposed that under any MANET with large number of mobile nodes the approach of multipath communication can address the load balancing more efficiently as compared to single path routing approach if there are huge number of routes utilized among all source and destination pairs.

In [12], the authors presented the performance evaluation multipath routing approach and reactive routing approach with load balancing technique.

In [13], author of this paper proposed method of load balancing in which number of realistic parameters like battery powers of every node, processing capabilities of every node, communication cost required for transferring the loads from overloaded nodes to under loaded mobile nodes.

In [14], author Saigal, et al. introduced another load balancing technique for MANET called as LARA (load aware routing in ad hoc). In this protocol, traffic density metric is utilized for presenting the contention degree at MAC layer. During the process of route setup, traffic density parameter is utilized for selecting the communication path with less traffic load.

In [15], the authors presented the details on selecting the right trade off among improved performance and increased routing overheads. 
In [16], authors present the new technique for achieving the both improved reliability in case of path failures as well as multipath based load balancing routing in MANETs. This paper achieves the objectives through full use of multiple paths in MANETs to solve frequent paths failures problems as well as load balancing problem.

In [17], authors Chakrabarti and Kulkarni present an approach for designing the alternate paths that are maintained as well as used in the DSR protocol. This method also provides the QoS solutions by ensuring the proper bandwidth for data transfer even if mobile nodes are under the mobility.

In [18], Souinli, et al. introduced another technique of loadbalancing which push data traffic from the network center. This approach delivered the new routing parameters which consider the mobile nodes centrality degree for reactive as well as proactive routing methods.

In [19], author Pham, et al. presented the multihop wireless communication networks in which IGW (internet gateway) method is used for providing the internet connectivity, wireless network linking with global Internet. However, for taking the benefits of capacity generated through the multiple gateways, routing protocol presented in [19] is required to balance the load efficiently between all the available IGWs in order to achieve the optimized network performance.

In [20], the authors Yoo, et al. introduced the load balancing technique called SLBA means simple load balancing approach. This method can easily added to any existing routing protocols (reactive only). This SLBA method reduces the traffic concentration by enabling every node for dropping the RREQ packets or for giving up the packet forwarding.

In [21], author Khamayseh, et al. presented a novel MLR (Mobility and Load aware Routing) method for reducing the impacts of broadcasting problem. Flooding process is controlled by MLR method by restricting messages of rebroadcast based on less speed as well as less loaded mobile nodes. In this method every mobile node takes decision on received RREQ message depending on number of parameters such as routing load, speed etc.

In [22], authors Cheng, et al. presented approach for formulating problem of dynamic load balanced clustering into the problem of dynamic optimization. To solve this problem, author presented the different types of dynamic genetic algorithms in MANETs.

\section{PROPOSED METHODOLOGY}

The flowchart in Figure 1 is showing the simulation work flow and comparative study parameters. To address the problem of achieving both efficient load balancing and energy efficiency we design and proposed novel algorithm called EELAR (energy efficient load aware routing) in which both factors traffic on mobile nodes and energy level of mobile nodes considered while communication. Algorithm 1 is our proposed algorithm.

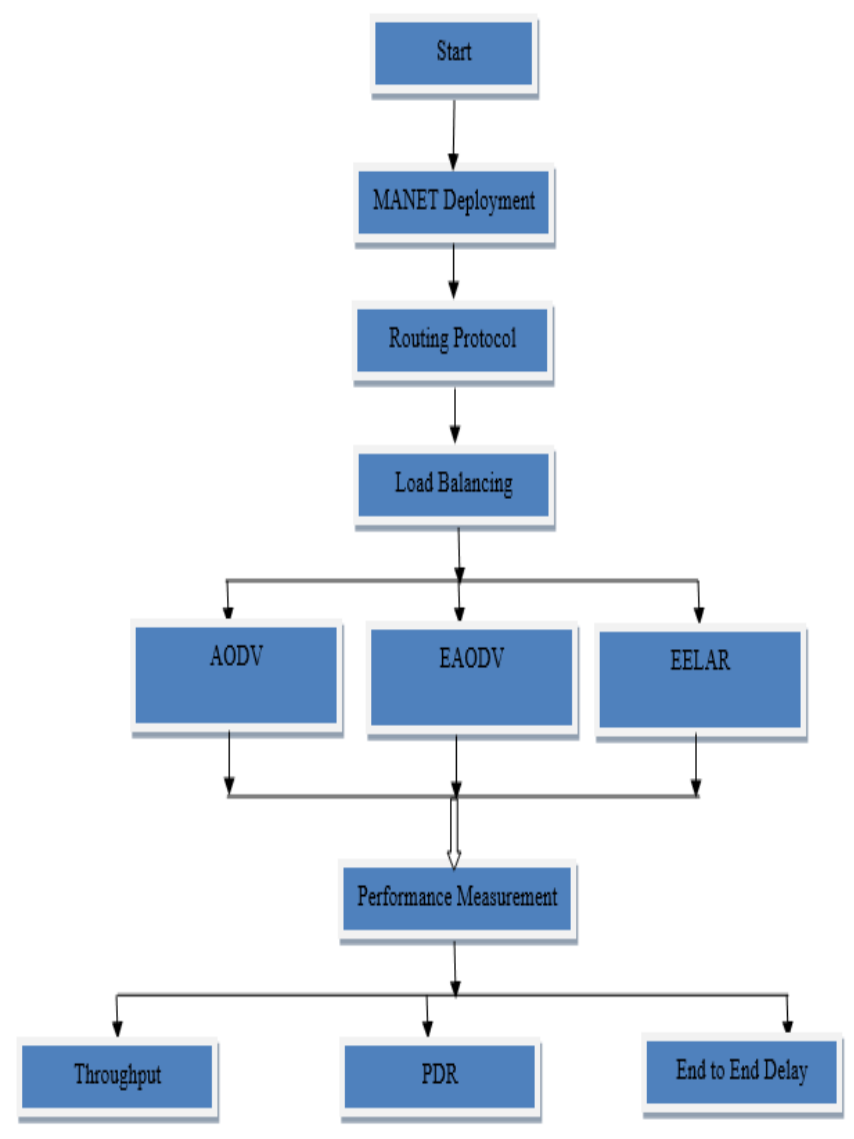

Fig. 1. Flowchart of simulation work

Algorithm 1: EELAR Method

Inputs:

Routing Table Entry,

packet $\mathrm{p}$,

node ID,

Set energy threshold,

Set load threshold values.

Step 1: Extract the current packet details

Step 2: Define the routing table pointer

Step 3: Extracting the Destination Area (DA) by computing the depth from sink node

Step 4: Extracting the Forwarding Area (FA) by computing the sink node neighbours

Step 5: Finding the shortest path from source to destination

Step 6: Update routing table entries

Step 7: Apply Energy Efficiency function

7.1.: Before starting data transfer, convert all nodes except source node into the sleep state 
7.2. If source node is ready to send data on selected active paths, then convert all nodes into active state from sleep state.

Step 8: Apply Load balancing function for data forwarding

Step 9: If energy level of any node goes below threshold or load on node goes particular threshold, then finds another alternate path in order to balance load or improved the network lifetime performance.

Step 10: If any node detects all its lower depth nodes below current threshold value, then it calculates new threshold and, start sending data on those paths again.

Step 11: Repeat this process still to the simulation ends.

Step 12: Stop.

\section{EXPERIMENTAL RESULTS}

\section{A. Network Configurations}

For practical work analysis, we used Network Simulator (NS2). In NS2, we implemented and evaluated the proposed EELAR protocol for comparative study purpose against existing AODV and EAODV routing protocols. This simulation is done on Ubuntu operating system and using NS2.34 version. The performance of routing protocols evaluation is done based on various network scenarios and data communication approaches under the various network conditions. For this simulation study we have used two main parameters such as varying mobility speed and varying number of mobile nodes in network. We have designed two different network scenarios for evaluating the performance of proposed protocol which is named as EELAR. Tables 1 and 2 show the other configuration parameters used.

TABLE I. SIMULATION CONFIGURATION FOR SCENARIO 1-VARYING MOBILITY SPEED

\begin{tabular}{|l|l|}
\hline Number of Nodes & 50 \\
\hline Traffic Patterns & CBR (Constant Bit Rate) \\
\hline Network Size $\left(\mathrm{X}^{*} \mathrm{Y}\right)$ & $1000 \times 1000$ \\
\hline Simulation Time & $100 \mathrm{~s}$ \\
\hline $\begin{array}{c}\text { Transmission Packet } \\
\text { Rate }\end{array}$ & $10 \mathrm{~m} / \mathrm{s}$ \\
\hline Pause Time & $1.0 \mathrm{~s}$ \\
\hline Routing Protocol & AODV/EAODV/EELAR \\
\hline MAC Protocol & 802.11 \\
\hline Channel Data Rate & $11 \mathrm{Mbps}$ \\
\hline Mobility Speed & $10 \mathrm{~m} / \mathrm{s}$ to $50 \mathrm{~m} / \mathrm{s}$ \\
\hline
\end{tabular}

TABLE II. SIMULATION CONFIGURATION FOR SCENARIO 2-VARYING MOBILE NODES

\begin{tabular}{|l|l|}
\hline Number of Nodes & $20-100$ \\
\hline Traffic Patterns & CBR (Constant Bit Rate) \\
\hline Network Size $\left(\mathrm{X}^{*} \mathrm{Y}\right)$ & $1000 \times 1000$ \\
\hline Simulation Time & $25 \mathrm{~s}$ \\
\hline $\begin{array}{c}\text { Transmission Packet } \\
\text { Rate }\end{array}$ & $10 \mathrm{~m} / \mathrm{s}$ \\
\hline Pause Time & $1.0 \mathrm{~s}$ \\
\hline Routing Protocol & AODV/EAODV/EELAR \\
\hline MAC Protocol & 802.11 \\
\hline Channel Data Rate & $11 \mathrm{Mbps}$ \\
\hline Mobility Speed & $30 \mathrm{~m} / \mathrm{s}$ \\
\hline
\end{tabular}

\section{B. Simulation Results}

We have compared the performance of three routing protocols using three performance metrics such as AODV, EAODV and proposed EELAR technique for load balancing QoS performance.

Scenario 1 Results:

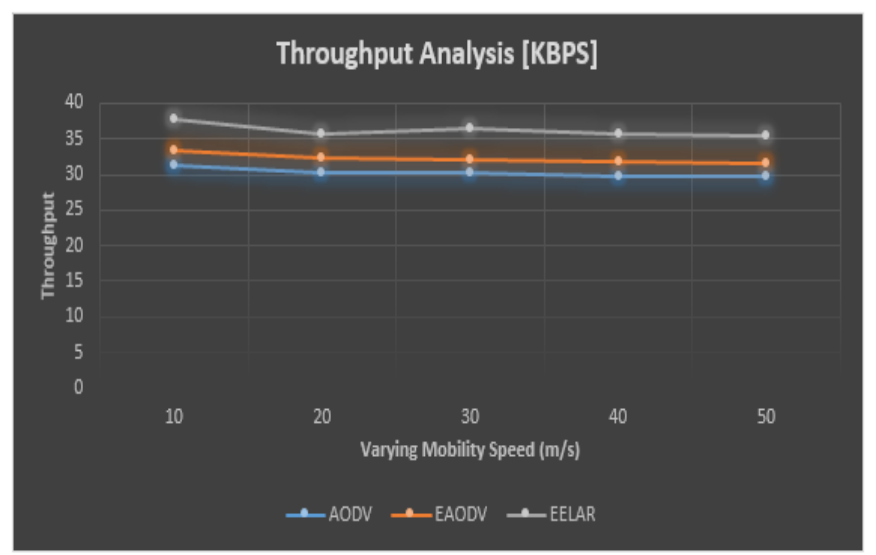

Fig. 2. Average throughput analysis for different load balancing methods of MANET

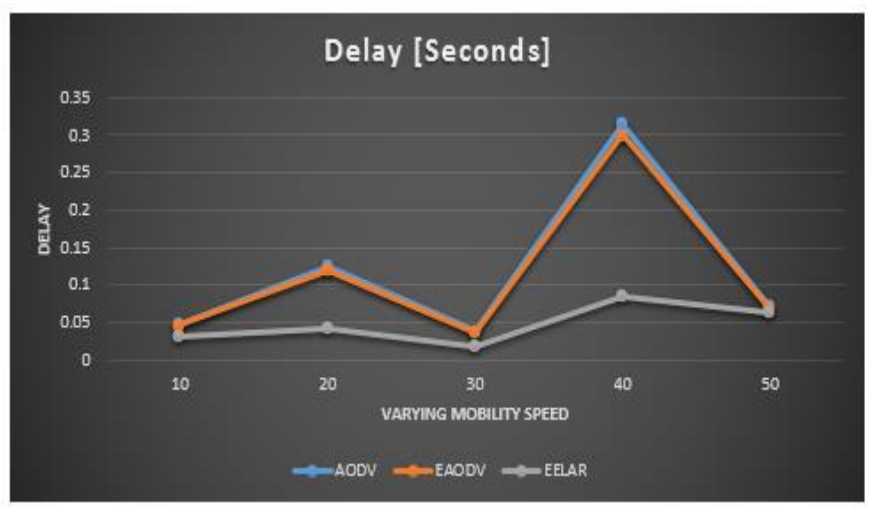

Fig. 3. Average delay analysis for different load balancing methods of MANET

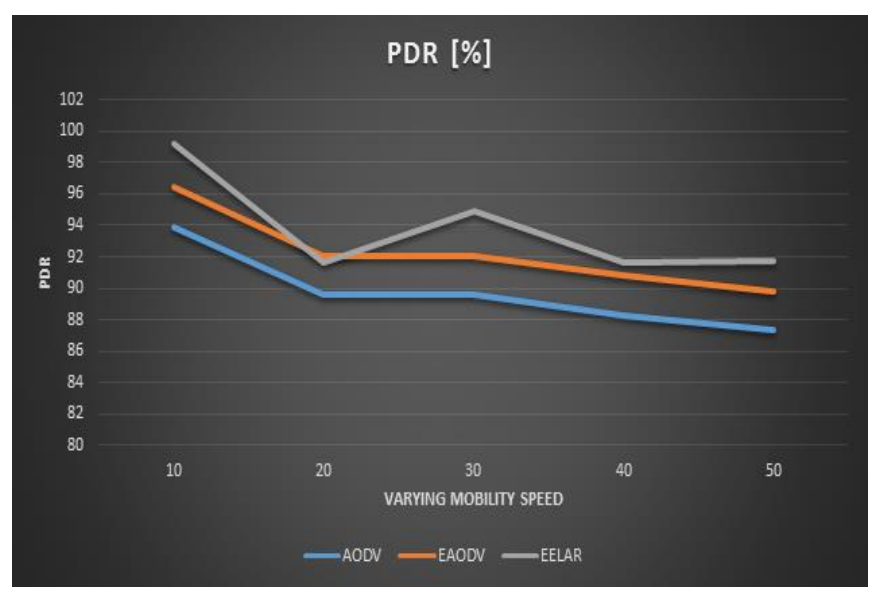

Fig. 4. Packet Delivery Ratio analysis for different load balancing methods of MANET 
Figures 2, 3, and 4 show the performance analysis for average throughput, average end to end delay, and packet delivery ratio, respectively for three studied routing protocols such as AODV, EAODV, and EELAR. We vary the mobility speed of mobile nodes by keeping total number of mobile nodes 50 to each mobility speed. The results show that we have achieved better QoS performance for proposed EELAR protocol. For this first scenario. it is showing that performance of throughput and PDR is improved by $35 \%$ as compared to EAODV protocol. The end to end delay is minimized by $15 \%$ to $18 \%$ as compared to EAODV protocol. Similarly, Figures 5, 6 , and 7 show the results for throughput, delay and PDR respectively for network scenario 2 . In the second scenario, the throughput shows an improvement of $38 \%$ and delay is minimized by $22 \%$ as compared to EAODV approach.

Scenario 2 Results

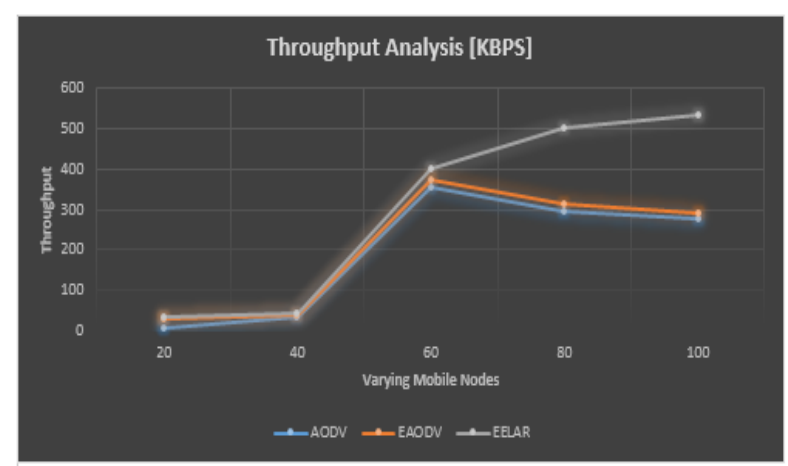

Fig. 5. Throughput analysis for different load balancing methods of MANET varying number of mobile nodes

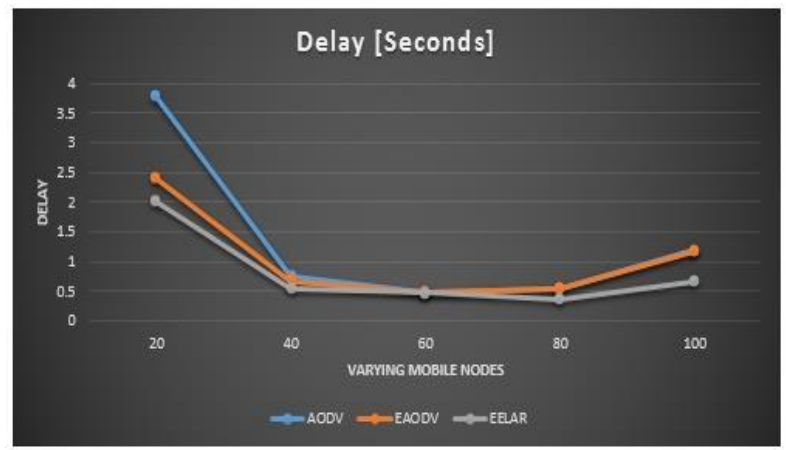

Fig. 6. Delay analysis for different load balancing methods of MANET varying number of mobile nodes

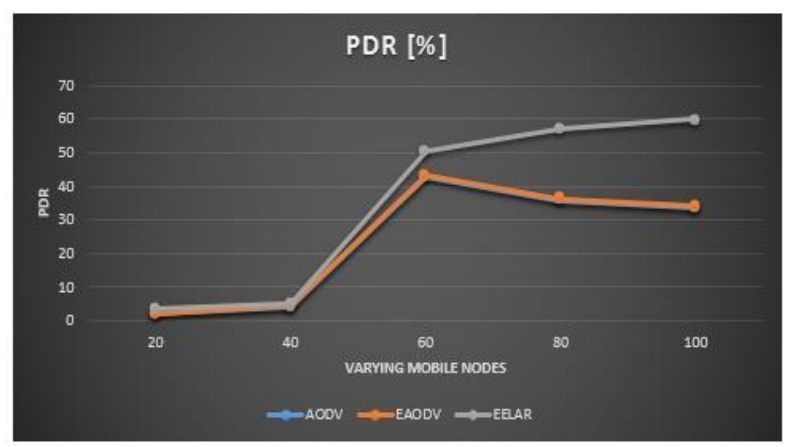

Fig. 7. Packet delivery ratio analysis for different load balancing methods of MANET varying number of mobile nodes

\section{CONCLUSION}

For MANET, load balancing technique plays a very vital role in order to achieve the QoS solutions. The traditional MANET routing protocols suffering from more routing overhead and decreased packet delivery ratio due to not addressing the load balancing in MANET communications. In this paper, we first presented the problems in MANET, then presented different solutions for load balancing techniques presented so far. We designed new load balancing technique for achieving the improved QoS performance as compared to existing EAODV and AODV routing protocols. The results section showing that we have simulated three protocols AODV, EAODV and proposed EELAR with two different network conditions. The results are compared by considering three important performance metrics of any routing protocol such as throughput, end to end delay and packet delivery ratio. In all cases, proposed load balancing approach shows improved performance when compared to existing methods.

\section{REFERENCES}

[1] S. Yin, X. Lin, MALB: MANET adaptive load balancing, in: IEEE Vehicular Technology Conference (VTC2004-Fall), vol. 4, September 2004, pp. 2843-2847.

[2] L. Zhang, Z. Zhao, Y. Shu, L. Wang, O.W., W. Yang, Load balancing of multipath source routing in ad hoc networks, in: Proceeding of the IEEE International Conference on Communications (ICC 2002), May 2002.

[3] K. Wu, J. Harms, Performance study of a multipath routing method for wireless mobile ad hoc networks, in: Proceeding of the Ninth International Symposium on Modeling, Analysis, and Simulation of Computer and Telecommunications Systems (MASCOTS'01), August 2001.

[4] L. Wang, L.F. Zhang, Y.T. Shu, M. Dong, O.W.W. Yang, Multipath source routing in wireless ad hoc networks, in: Proceeding of IEEE CCECE, 2000, p. 479.

[5] A. Valera, W. Seah, S.V. Rao, Cooperative packet caching and shortest multipath routing in mobile ad hoc networks, in: Proceeding of IEEE INFOCOM, 2003.

[6] S. Roy, S. Bandyopadhyay, T. Ueda, K. Hasuike, Multipath routing in ad hoc wireless networks with omni directional and directional antenna: a comparative study, in: Proceeding of the Fourth International Workshop on Distributed Computing, Mobile and Wireless Computing (IWDC), 2002, pp. 184-191.

[7] A. Zhou, H. Hassanein, Load-balanced wireless ad hoc routing, in: IEEE Canadian Conference on Electrical and Computer Engineering, vol. 2, 2001, pp. 1157-1161.

[8] Y.J. Lee, G.F. Riley, A workload-based adaptive load-balancing technique for mobile ad hoc networks, in: IEEE Wireless Communications and Networking Conference (WCNC'2005), vol. 1, 2005, pp. 2002-2007.

[9] M. Perlman, Z. Haas, P. Scholander, S. Tabrizi, Alternate path routing for load balancing in mobile ad hoc networks, in: IEEE Military Communications Conference (MILCOM 2000), October 2000.

[10] P.Pham, S. Perreau, Multi-path routing protocol with load balancing policy in mobile ad hoc networks, in: IFIP Int'l Conference on Mobile and Wireless Communications Networks (MWCN 2002), September 2002.

[11] Y. Ganjali, A.Keshavarzian, Load balancing in ad hoc networks: singlepath routing vs. multi-path routing, in: Twenty-third Annual Joint Conference of the IEEE Computer and Communications Societies (INFOCOM 2004), March 2004.

[12] P. Pham and S. Perreau, "Performance analysis of reactive shortest path and multi-path routing mechanism with load balance," IEEE Conference on Computer Communications (INFOCOM 2003), March 2003.

[13] Turgut, D.; Turgut, B.; Das, S.K.; Elmasri, R.; , "Balancing loads in mobile ad hoc networks," Telecommunications, 2003. ICT 2003, 10th 
International Conference on , vol.1, no., pp. 490- 495 vol.1, 23 Feb.-1 March 2003.

[14] V. Saigal, A. Nayak, S. Pradhan, and R. Mall, "Load balanced routing in mobile ad hoc networks", Computer Communications, Vol.27, 2004, pp.295-305.

[15] Peter P.Pham, Sylvie Perreau , "Increasing the network performance using multi-path routing mechanism with load balance, Ad Hoc Networks, Volume 2, Issue 4, October 2004, Pages 433-459.

[16] Antonios Argyriou, Vijay Madisetti, "Using a new protocol to enhance path reliability and realize load balancing in mobile ad hoc networks", Ad Hoc Networks, Volume 4, Issue 1, January 2006, Pages 60-74.

[17] Gautam Chakrabarti, Sandeep Kulkarni, "Load balancing and resource reservation in mobile ad hoc networks", Ad Hoc Networks, Volume 4, Issue 2, March 2006, Pages 186-203.

[18] [Oussama Souihli, Mounir Frikha, Mahmoud Ben Hamouda, "Loadbalancing in MANET shortest-path routing protocols", Ad Hoc Networks, Volume 7, Issue 2, March 2009, Pages 431-442.
[19] Vinh Pham ,Erlend Larsen ,Paal E. Engelstad, Øivind Kure, "Performance analysis of gateway load balancing in ad hoc networks with random topologies ", Proceedings of the 7th ACM international symposium on Mobility management and wireless access, 2009, pp.6674.

[20] Younghwan Yoo, Sanghyun Ahn, Dharma P. Agrawal, "Impact of a simple load balancing approach and an incentive-based scheme on MANET performance", Journal of Parallel and Distributed Computing, Volume 70, Issue 2, February 2010, Pages 71-83.

[21] Yaser Khamayseh, Ghadeer Obiedat, Munner Bani Yassin, "Mobility and Load aware Routing protocol for ad hoc networks", Journal of King Saud University - Computer and Information Sciences, Volume 23, Issue 2, July 2011, Pages 105-113.

[22] Hui Cheng, Shengxiang Yang, Jiannong Cao, "Dynamic Genetic Algorithms for the Dynamic Load Balanced Clustering Problem in Mobile Ad Hoc Networks", Expert Systems with Applications, , 5 September 2012. 